wurde die Debatte über den Jugendmedienschutz meist darauf reduziert, Chancen und Risiken gegeneinander abzuwägen. Dieser Blick erweist sich als zu eng. Es kann nicht mehr nur um die Einschätzung gehen, dass das eine verliert, wenn das andere gewinnt. Den Ausgangspunkt für ein aus der Perspektive des Jugendmedienschutzes gutes Internet für Kinder bildet das Kind selbst. Es sind seine Praktiken, seine Motive, Erfahrungen und Umgangsformen bei der Nutzung digitaler Medien, die die Richtungen anzeigen. Es vollzieht sich dabei der Umgang mit digitalen Medien für Kinder und Jugendliche nicht isoliert im medialen Raum, sondern er ist eingebettet in ein Leben in familiären, sozialen, kulturellen Bezügen. Digitaler Jugendschutz ist insofern kein Klotz am Bein, sondern ein ins Netz ragender Arm, der Hilfestellung für Heranwachsende und deren Familien beim Aufwachsen mit dem Internet anbietet. Das Buch leistet auf Grundlage von Forschung einen Beitrag dafür, wie wir als internationale Gesellschaft besser zusammenfinden können, um diese Aufgabe anzugehen.

Friederike Siller

Ingrid Paus-Hasebrink / Sascha Trültzsch / Alois Pluschkowitz / Christine W. Wijnen (Hrsg.)

Integrative AV- und OnlineKommunikationsforschung

Perspektiven - Positionen - Projekte

Baden-Baden: Nomos, 2013. - 238 S.

ISBN 978-3-8487-0310-4

(Lebensweltbezogene Medienforschung:

Angebote - Rezeption - Sozialisation; 1)

Das Autor(inn)enteam lehrt und forscht in Salzburg und Wien und veröffentlicht mit diesem Buch den zweiten „Grundlagenband der Abteilung für Audiovisuelle und Online-Kommunikation am Salzburger Fachbereich Kommunikationswissenschaft" (S. 14). Die erste Publikation präsentierte eine „Einführung in die Audiovisuelle Kommunikation" (Paus-Hasebrink u. a. 2006). Die nun vorliegende Arbeit widmet sich dem Anliegen, eine integrative AV- und Online-Kommunikationsforschung vorzustellen und $\mathrm{zu}$ diskutieren. Angesichts einer radikal veränderten Medienlandschaft (Stichwort Digitalisierung von öffentlicher und privater Kommunikation) stellen sich die $\mathrm{Au}-$ tor(inn)en der Herausforderung, in theoretischer wie auch methodischer Perspektive eine gegenstandsangemessene Medienforschung zu konzipieren und in Forschungsprojekten zu erproben.

Sie gehen in folgenden Schritten vor: In Kapitel 2 „Positionen der Kommunikationswissenschaft" wird „eine Standortbestimmung des Feldes der Audiovisuellen und Online-Kommunikation" (S.14) in der medien- und kommunikationswissenschaftlichen Forschung vorgenommen. Das Autor(inn)enteam wählt hier einen, wie sie meinen „ungewöhnlichen Weg“ (ebd.), indem sie sieben ausgewiesene Kommunikationswissenschaftler(inn)en um ihre Antworten bitten. Die Experteninterviews wurden mit Sonia Livingstone, Nico Carpentier, Uwe Hasebrink, Andreas Hepp, David Smahel, Gerit Götzenbrucker und Lothar Mikos geführt und sollen für den Leser ein „,internationales Stimmungsbild zeichnen“" (ebd.). Sascha Trültzsch und Alois Pluschkowitz fassen die Ergebnisse dieser diskursiven Standortbestimmung der Kommunikationswissenschaft in Kapitel 2.8 knapp zusammen.

Daran schließt das von Paus-Hasebrink verfasste theorie-orientierte Kapitel 3 „Audiovisuelle und Online-Kommunikation - Theoretische Wege zur Analyse der komplexen Zusammenhänge von Produktions-, Angebots- und Aneignungsweisen " an: Thematisiert werden darin die technischen und kulturellen Veränderungen, die sich mit der fortschreitenden $\mathrm{Me}$ diatisierung, Digitalisierung und Globalisierung der Lebenswelten der Menschen vor allem auch für das Feld der Individualkommunikation bereits bis heute ergeben haben. Diese Wandlungsprozesse stellen die bisher gültigen Kommunikationstheorien vor gravierende Probleme, die nur im Rahmen einer integrativen Theoriebildung gelöst werden können. PausHasebrink schreibt: „Zentrales Anliegen ist die Integration verschieden gelagerter, bisher wenig zueinander in Beziehung gesetzter Theorietraditionen, die ein ganzheitliches, lebensweltorientiertes Verständnis von Audiovisueller und Online-Kommunikation ermöglichen" (S. 15). Die Autorin orientiert sich insbesondere an „weiterentwickelten sozialphänomenologischen bzw. sozialkonstruktivistischen Lebensweltkonzeptionen" (S. 75) sowie am Ansatz der „Lebensführung“ (S. 77ff.) bzw. der „Lebensaufgaben“ (S. 85ff.). „Ziel (...) ist es - kurz gesagt -, eine theoretische Basis für eine integrative, molare - das heißt auch sozialökologische - Mehr-Ebenen-Analyse von Prozessen der AV- und Online-Kommunikation zu legen“" (S. 61).

In Kapitel 4 „Methodenvielfalt in der Forschung zu Audiovisueller und Online-Kommunikation“ (S. 100ff.) werden von den Au- 
tor(inn)en Trültzsch, Wijnen und Dürager zentrale Methoden der Produktions-, Produkt-, Nutzungs- und Rezeptionsanalyse vorgestellt, die jeweils in integrativer Perspektive zur Anwendung gebracht werden sollen. Im anschließenden Kapitel 5 „Beispiele integrativer AVund Onlineforschung" (S. 148ff.) stellen wechselnde Autor(inn)enteams bereits abgeschlossene sowie noch laufende Forschungsprojekte am Salzburger Fachbereich Kommunikationswissenschaft vor. Besprochen werden die unterschiedlich komplexen Forschungsprojekte: Mediensozialisationsforschung, Medienkindheit-Markenkindheit, Heranwachsen mit dem Social Web, Kinderfernsehen, Musikfernsehen und Reality TV, Forschungen für die Medienpraxis, berufsfeldorientierte studentische LehrLern-Medienprojekte sowie das zukünftige Projekt zum Thema „Dead Drops“. Das Buch schließt mit einer Schlusszusammenfassung (S. 236ff.)

Die Publikation ist sinnvoll konzipiert und klar verständlich. Der/die Leser/in erhält einen guten Zugang zu dem in Salzburg vertretenen Ansatz einer lebensweltbezogenen Medienforschung. Das Zielpublikum ist für den Verfasser dieser Rezension eher ein studentisches Publikum, dem ein interessantes, gut lesbares Lehrbuch angeboten wird: Kurzweilig zu lesende Experteninterviews sind mit theorie-orientierten Klärungen verbunden, die erweitert werden durch methodologisch-methodische Ausführungen, die eine Konkretisierung durch die Präsentation von beispielhaften Medienforschungen erfahren. Das Buch stellt die Arbeit des Lehrstuhls Paus-Hasebrink am Fachbereich Kommunikationswissenschaft der Universität Salzburg in umfassender Weise vor und vermittelt auch einen guten Überblick über das nähere fachliche Netzwerk dieser Arbeitsgruppe. Der neu herausgegebenen Buchreihe ist $\mathrm{zu}$ wünschen, dass in ihr recht bald auch Forschungsarbeiten von anderen Arbeitsgruppen an anderen Universitäten veröffentlicht werden, die die Reichweiten und Grenzen dieses Konzepts einer integrativen lebensweltbezogenen Medienforschung in Theorie und Praxis weiter zu bestimmen helfen.

Klaus Neumann-Braun

\section{Colin Porlezza}

\section{Gefährdete journalistische Unabhängigkeit}

Zum wachsenden Einfluss von Werbung auf redaktionelle Inhalte

Konstanz, München: UVK, 2014. - 289 S.

ISBN 978-3-86764-461-7

$\mathrm{Zu}$ den normativen Funktionsbestimmungen des Journalismus zählt der Anspruch, unbeeinflusst von kommerziellen Interessen und insofern aus unabhängiger Sicht zu berichten. Wie weit folgt der real stattfindende, mehr oder weniger über Werbeerlöse gegenfinanzierte Journalismus diesem Erfordernis? Seit den weitreichenden Studien von Karl Bücher zu Beginn des 20. Jahrhunderts stellt sich die Frage nach dem Einfluss der Anzeigen auf den redaktionellen Inhalt, ein Thema, das schon viele Medienforscher umgetrieben hat und bis heute strittig diskutiert wird. Dies liegt auch daran, dass ein Nachweis der inhaltlichen Einflussnahme durch Anzeigenkunden meist nur okkasionell, quasi als Ausnahme von der Regel nachgewiesen werden konnte, nicht aber strukturell - was die These erlaubt, dass es diese strukturelle $\mathrm{Ab}$ hängigkeit vielleicht nicht gibt. Hier spätestens stellt sich die Frage, um welchen Journalismus es dabei gehen solle. Dass viele Unterhaltungsmagazine und Anzeigenblätter Werbebotschaften redaktionell camouflieren, wissen auch große Teile des Publikums, sie lohnen keinen wissenschaftlichen Nachweis. Und wie verhält es sich mit den kostenlos dargebotenen, d. h. komplett über Werbung finanzierten Tageszeitungen, den sog. Pendlerzeitungen, eine Gattung, die es insbesondere in der Schweiz und in Österreich gibt?

Der Buchtitel verspricht Erkenntnisse über „den Einfluss der Werbung auf redaktionelle Inhalte“ und Aussagen über die „journalistische Unabhängigkeit“. Davon kann indessen nicht die Rede sein. Die Studie befasste sich vielmehr mit diesen Gratisblättern in der Schweiz, die als informatorische Unterhaltungslektüre für Berufspendler auf dem Weg zur Arbeit (bzw. für den Nachhauseweg zum Feierabend) konzipiert sind, die in 15 bis 20 Minuten rezipiert werden sollen und die keineswegs dieselbe Informationsleistung erbringen wie eine Bezahlzeitung. Trotz der enormen Reichweite, die vor allem das Blatt „20 Minuten" in der Schweiz erzielt, ist fraglich, ob die Nutzer dem Blatt vertrauen bzw. aufgrund der Finanzierung über Produktanzeigen entsprechend zurückhaltend mit der Produkt- und Wirtschaftsberichterstattung umgehen. 